\title{
Analysis of Timing Jitter for Ultrashort Soliton Communication Systems Using Perturbation Methods
}

\author{
Margarida FACÃO and Mário FERREIRA \\ Universidade de Aveiro, Campus de Santiago, 3810 Aveiro, Portugal
}

\begin{abstract}
We analyse timing jitter of ultrashort soliton systems taking into account the major higher order effects, namely, intrapulse Raman scattering and third order dispersion and using adiabatic perturbation theory. We obtain an expression for the soliton arrival time variance that depends on the quintic power of propagation distance. This timing jitter could be partly overcome by use of bandwidth-limited amplification as it happens in systems using longer solitons. In this case the quintic dependence with distance is reduced to a linear one.
\end{abstract}

\section{Introduction}

In soliton communication systems, the transmission capacity could be improved using picosecond-subpicosecond solitons. In such regime the major higher order effects are intrapulse Raman scattering (IRS) and third order dispersion (TOD). The former effect causes a soliton self-frequency shift (SSFS) and increases the timing jitter.

Taking the effects above into account, the equation that describes the propagation of pulses in nonlinear and dispersive optical fibers with amplification is the following

$$
i q_{Z}+\frac{1}{2} q_{T T}+|q|^{2} q=i \rho_{0} q+T_{R} q|q|_{T}^{2}+i \rho_{3} q_{T T T}
$$

where $q=A \sqrt{\gamma \tau_{0}^{2} /\left|\beta_{2}\right|}, Z=\left|\beta_{2}\right| z / \tau_{0}^{2}$ and $T=\tau / \tau_{0}$ are normalized quantities for the complex amplitude $A$ of the pulse envelope, the propagation distance $z$ and the time $\tau$ of a referential that moves with group velocity, respectively. $\beta_{2}$ is the group-velocity dispersion coefficient, $\gamma$ is a nonlinear parameter and $\tau_{0}$ is the pulse width. $\rho_{0}=G-\Gamma$ is the excess gain where $\Gamma=\alpha \tau_{0}^{2} / 2\left|\beta_{2}\right|$ and $G=g \tau_{0}^{2} /\left|\beta_{2}\right|$ are normalized parameters for the absorption coefficient, $\alpha$, and the gain coefficient, $g$, respectively. $T_{R}=\tau_{R} / \tau_{0}$ and $\rho_{3}=\beta_{3} / 6\left|\beta_{2}\right| \tau_{0}$ are as well normalized parameters for Raman gain curve parameter, $\tau_{R}$, and third order dispersion coefficient, $\beta_{3}$, respectively.

When the right hand member of equation (1) is zero the equation becomes the nonlinear Schrödinger equation (NLSE). It is sufficient to describe several picosecond pulse propagation in lossless fibers, as it was shown by Hasegawa and Tappert [1]. That equation supports soliton solutions and it can be solved using the inverse scattering method.

For non-zero but small right hand member, the solutions can be drawn using NLSEsolitons and a perturbation method. The singular NLSE-soliton is given by

$$
q(T, Z)=\eta(Z) \operatorname{sech}\left[\eta(Z)\left(T-T_{0}(Z)\right] \exp [-i \kappa(Z) T+i \varsigma(Z)]\right.
$$


and it is a good zero-order approximated solution [2]. For adiabatic perturbations, only soliton parameters, namely: amplitude, $\eta$, frequency, $\kappa$, position, $T_{0}$ and phase, $\varsigma$, are affected and no radiation or solitons tails are created.

In this work we apply perturbation theory to analyse both timing jitter of ultrashort solitons and its control by use of bandwidth-limited amplification.

\section{Timing jitter of ultrashort solitons}

Coherent amplification is always accompanied by generation of spontaneous-emission noise. Because of nonlinearity, this noise have not only an additive behaviour but induces a change of amplitude $\delta \eta$, timing $\delta T_{0}$, frequency $\delta \kappa$ and phase $\delta \varsigma$. These perturbations are uncorrelated with zero averages and variances given by $[3,4]$

$$
\begin{aligned}
& \left\langle\delta \eta^{2}\right\rangle=\frac{G-1}{N_{0}} \eta, \quad\left\langle\delta \kappa^{2}\right\rangle=\frac{G-1}{3 N_{0}} \eta, \\
& \left\langle\delta T_{0}^{2}\right\rangle=\frac{\pi^{2}}{12 \eta} \frac{G-1}{N_{0}} \quad \text { and } \quad\left\langle\delta \varphi^{2}\right\rangle=\frac{1}{3 \eta}\left(\frac{\pi^{2}}{12}+1\right) \frac{G-1}{N_{0}},
\end{aligned}
$$

where $N_{0}=E_{s} / 2 \hbar \omega$ ( $\omega$ is the central frequency of the pulse) is the number of photons per bit of information and the gain is $G=\exp \left(\alpha z_{a}\right)$, where $z_{a}$ is the amplifier distance.

For longer solitons (several picoseconds), only the random change of frequency (and thus of group velocity) modifies the arrival timing of the soliton. This phenomenon is known as the Gordon-Haus effect [3]. Taking into account all the perturbations induced by each amplifier stage, we obtain an ultimate expression for the timing variance that grows with the cubic power of $Z$

$$
\left\langle\delta T_{0}^{2}(Z)\right\rangle=\frac{(G-1) \eta}{9 N_{0}} \frac{Z^{3}}{Z_{a}}
$$

where $Z_{a}$ is the normalized amplifier distance.

Considering that the gain compensate exactly the loss, i.e., $\Gamma=G$ in equation 1 , and applying adiabatic perturbation theory to the resultant equation, we get the following parameter evolution equations

$$
\begin{aligned}
& \frac{d \eta}{d Z}=0, \\
& \frac{d \kappa}{d Z}=-\frac{8}{15} T_{R} \eta^{4}, \\
& \frac{d T_{0}}{d Z}=-\kappa+\rho_{3}\left(\eta^{2}+3 \kappa^{2}\right) .
\end{aligned}
$$

From (6) we have the evolution of $\kappa$ given by

$$
\kappa(Z)=-\frac{8}{15} T_{R} \eta_{0}^{4} Z+\kappa_{0}
$$

and from (7)

$$
\begin{aligned}
T_{0}(Z)= & \frac{4}{15} T_{R} \eta_{0}^{4} Z^{2}-\kappa_{0} Z+\rho_{3} \eta_{0}^{2} Z \\
& +\frac{64}{225} \rho_{3} T_{R}^{2} \eta_{0}^{8} Z^{3}-\frac{8}{5} \rho_{3} T_{R} \eta_{0}^{4} \kappa_{0} Z^{2}+3 \rho_{3} \kappa_{0}^{2} Z+T_{0 i} .
\end{aligned}
$$



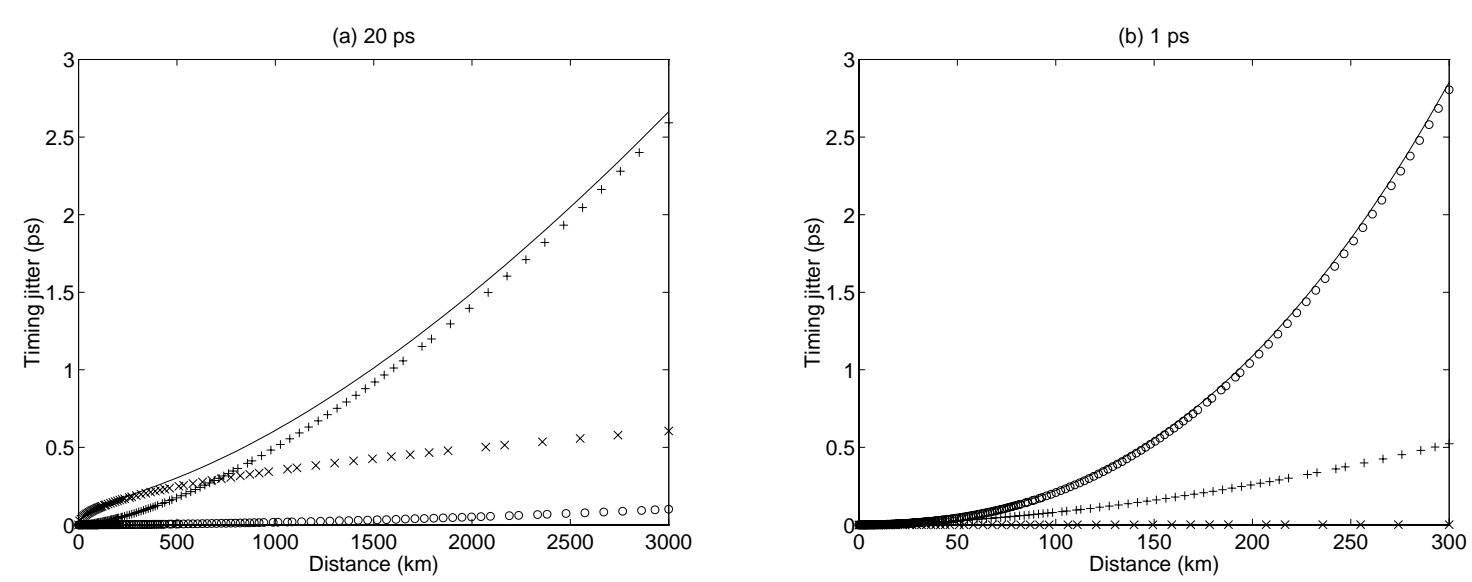

Figure 1. Growth of timing jitter with real distance. Different kind of contributions: (ooo) amplitude fluctuations; $(+++)$ frequency fluctuations; $(x x x)$ position fluctuations and $(-)$ total timing jitter. For both graphics amplifier spacing is $80 \mathrm{~km}, N_{0}=8.38 \times 10^{-6} / \tau_{s} \mathrm{~s}^{-1}, \alpha=0.046$ $\mathrm{km}^{-1}, \beta_{2}=-0.5 \mathrm{ps}^{2} \mathrm{~km}^{-1}, \beta_{3}=0.05 \mathrm{ps}^{3} \mathrm{~km}^{-1}$ and $\tau_{R}=6$ fs. The full width at half maximum is (a) $\tau_{s}=20 \mathrm{ps}$, (b) $\tau_{s}=1 \mathrm{ps}$.

The change in timing after one amplifier stage localized at $Z_{n}=n Z_{a}$ is obtained by differentiation of equation (9) and is given by [5]

$$
\begin{aligned}
\delta T_{0}\left(Z_{n}\right)= & \left(\frac{16}{15} T_{R} \eta_{0}^{3} Z_{n}^{2}+2 \rho_{3} \eta_{0} Z_{n}+\frac{512}{225} \rho_{3} T_{R}^{2} \eta_{0}^{7} Z_{n}^{3}-\frac{32}{5} \rho_{3} T_{R} \eta_{0}^{3} \kappa_{0} Z_{n}^{2}\right) \delta \eta\left(Z_{n}\right) \\
& +\left(-\frac{8}{5} \rho_{3} T_{R} \eta_{0}^{4} Z_{n}^{2}+6 \rho_{3} \kappa_{0} Z_{n}-Z_{n}\right) \delta \kappa\left(Z_{n}\right)+\delta T_{0 i}\left(Z_{n}\right) .
\end{aligned}
$$

The variance of arrival timing after $N=Z / Z_{a}$ amplifiers stages are obtained by summation of the uncorrelated $\delta T_{0}$, and we obtain

$$
\begin{aligned}
\left\langle\delta T_{0}^{2}(Z)\right\rangle= & \left(\frac{256}{1125} T_{R}^{2} \eta_{0}^{6} \frac{Z^{5}}{Z_{a}}+\frac{4}{3} \rho_{3}^{2} \eta_{0}^{2} \frac{Z^{3}}{Z_{a}}+\frac{16}{15} \rho_{3} T_{R} \eta_{0}^{4} \frac{Z^{4}}{Z_{a}}\right)\left\langle\delta \eta^{2}\right\rangle \\
& +\left(\frac{2}{5} \rho_{3} T_{R} \eta_{0}^{4} \frac{Z^{4}}{Z_{a}}+\frac{1}{3} \frac{Z^{3}}{Z_{a}}\right)\left\langle\delta \kappa^{2}\right\rangle+\frac{Z}{Z_{a}}\left\langle\delta T_{0 i}^{2}\right\rangle,
\end{aligned}
$$

where we have replaced the summation by an integration. As we can see, by inspection of the above expression, for large distances the timing jitter grows with $Z^{5}$. This dependence comes from amplitude fluctuations through IRS effect.

Fig. 1 shows timing jitter, $\sqrt{\left\langle\delta T_{0}^{2}\right\rangle}$, as function of distance for two different soliton durations, $\tau_{s}=20 \mathrm{ps}$ and $\tau_{s}=1 \mathrm{ps}$. We can see that, for the longer soliton, timing jitter comes particularly from frequency fluctuations. However, for the shorter soliton, the contribution of amplitude fluctuations dominates.

Fig. 2 shows timing jitter growing with the amplifier spacing for $\tau_{s}=1 \mathrm{ps}$. We can see that the amplitude fluctuations contribution dominates over the others contributions.

\section{Jitter control by bandwidth-limited amplification}

The spectral filtering technique have been proposed to control the Gordon-Haus jitter of longer solitons. It has been shown that this technique can reduce the cubic dependence 


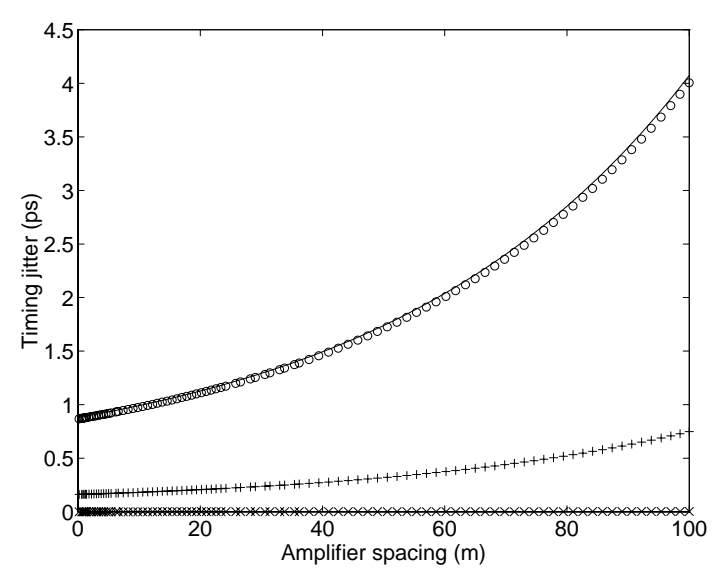

Figure 2. Growth of timing jitter with amplifier spacing, where the propagation distance is 300 km. Different kind of contributions: (ooo) amplitude fluctuations; $(+++)$ frequency fluctuations; (xxx) position fluctuations and (-) total timing jitter. $N_{0}=8.38 \times 10^{-6} / \tau_{s} \mathrm{~s}^{-1}, \alpha=0.046 \mathrm{~km}^{-1}$, $\beta_{2}=-0.5 \mathrm{ps}^{2} \mathrm{~km}^{-1}, \beta_{3}=0.05 \mathrm{ps}^{3} \mathrm{~km}^{-1}, \tau_{R}=6$ fs and $\tau_{s}=1 \mathrm{ps}$.

with distance, characteristic of Gordon-Haus effect, to a linear dependence [6]. In ultrashort soliton regime the proper finite optical gain bandwidth of the amplifier can act as a filter and it can be efficient to control timing jitter and soliton self frequency shift.

The propagation equation for ultrashort solitons that includes bandwidth-limited amplification is given by

$$
i q_{Z}+\frac{1}{2} q_{T T}+|q|^{2} q=i \rho_{0} q+i \rho_{2} q_{T T}+T_{R} q|q|_{T}^{2}
$$

where $\rho_{2}=-1 / 2\left|\beta_{2}\right| \partial^{2} g / \partial \omega^{2}$ is the filtering coefficient. In this case $\rho_{0}$ must be different of zero to compensate for the loss that solitons suffer at wings of their spectrum. We have neglected TOD term as we have shown that the main contribution to the jitter comes from IRS. Applying adiabatic perturbation theory we obtain the following parameter evolution equations

$$
\begin{aligned}
& \frac{d \eta}{d Z}=2 \rho_{0} \eta-2 \rho_{2} \eta\left(\frac{\eta^{2}}{3}+\kappa^{2}\right) \\
& \frac{d \kappa}{d Z}=-\frac{4}{3} \rho_{2} \eta^{2} \kappa-\frac{8}{15} T_{R} \eta^{4} \\
& \frac{d T_{0}}{d Z}=-\kappa
\end{aligned}
$$

The first two equations have an equilibrium point $\left(\eta_{e}, \kappa_{e}\right)=\left(1,-2 T_{R} / 5 \rho_{2}\right)$ [7]. The linearized equations around this point become

$$
\begin{aligned}
& \frac{d \eta}{d Z}=\frac{16}{25} \frac{T_{R}^{2}}{\rho_{2}}+\frac{4 \rho_{2}}{3}(1-\eta)+\frac{8}{5} T_{R} \kappa, \\
& \frac{d \kappa}{d Z}=\frac{8}{15} T_{R}-\frac{16}{15} T_{R} \eta-\frac{4}{3} \rho_{2} \kappa .
\end{aligned}
$$




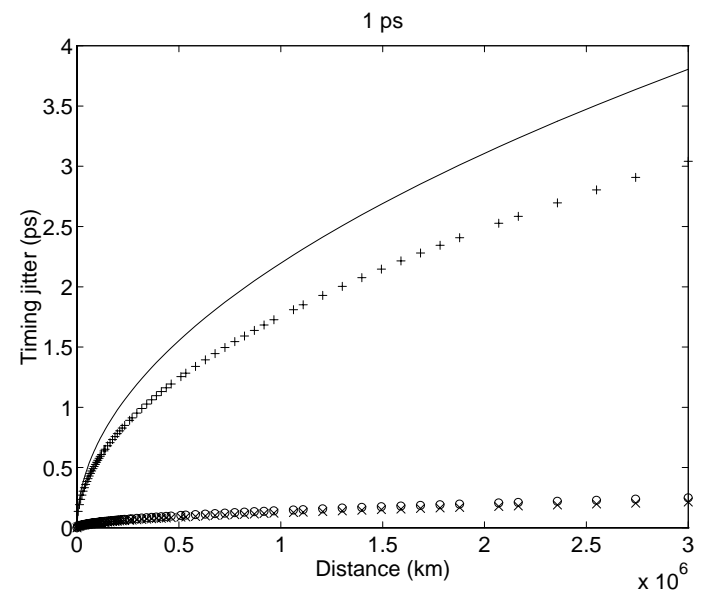

Figure 3. Growth of timing jitter with real distance in presence of bandwidth-limited amplification. Different kind of contributions: (ooo) amplitude fluctuations; $(+++)$ frequency fluctuations; ( $\mathrm{xx}$ ) position fluctuations and (-) total timing jitter. $z_{a}$ is $80 \mathrm{~km}, N_{0}=8.38 \times 10^{-6} / \tau_{\mathrm{s}} \mathrm{s}^{-1}$, $\alpha=0.046 \mathrm{~km}^{-1}, \beta_{2}=-0.5, \rho_{2}=0.03 \mathrm{ps}^{3} \mathrm{~km}^{-1}, \tau_{R}=6$ fs and $\tau_{s}=1 \mathrm{ps}$.

These equations can be solved exactly and by equation (15) we have

$$
\begin{aligned}
& T_{0}(Z)=\frac{-1}{20\left(25 \rho_{2}^{3}+24 \rho_{2} T_{R}^{2}\right)}\left(375 \rho_{2} \kappa_{0}+50 \rho_{2} T_{R}\left(2+\eta_{0}\right)-8 T_{R} Z\left(25 \rho_{2}^{2}+24 T_{R}^{2}\right)\right) \\
& +\frac{\exp \left(-\frac{4 \rho_{2} Z}{3}\right)}{120\left(25 \rho_{2}^{3}+24 \rho_{2} T_{R}^{2}\right)}\left(150 \rho_{2}\left(15 \kappa_{0} \rho_{2}+2 T_{R}\left(2+\eta_{0}\right) \cos \left(\frac{8}{5} \sqrt{\frac{2}{3}} T_{R} Z\right)\right)\right. \\
& \left.+5 \sqrt{6}\left(25 \rho_{2}^{2}\left(\eta_{0}-1\right)-180 \kappa_{0} \rho_{2} T_{R}-72 T_{R}^{2}\right) \sin \left(\frac{8}{5} \sqrt{\frac{2}{3}} T_{R} Z\right)\right)+T_{0 i} .
\end{aligned}
$$

Analogously to section 2 we differentiate equation (18) to obtain the change in timing after one amplifier stage localized at $Z_{n}=n Z_{a}$

$$
\begin{aligned}
& \delta T_{0}\left(Z_{n}\right)=\frac{-60 T_{R}+\exp \left(-\frac{4 \rho_{2} Z_{n}}{3}\right)\left(60 T_{R} \cos \left(\frac{8}{5} \sqrt{\frac{2}{3}} T_{R} Z_{n}\right)+25 \sqrt{6} \rho_{2} \sin \left(\frac{8}{5} \sqrt{\frac{2}{3}} T_{R} Z_{n}\right)\right)}{24\left(25 \rho_{2}^{2}+24 T_{R}^{2}\right)} \delta \eta_{0} \\
& +\frac{-75 \rho_{2}+\exp \left(-\frac{4 \rho_{2} Z_{n}}{3}\right)\left(75 \rho_{2} \cos \left(\frac{8}{5} \sqrt{\frac{2}{3}} T_{R} Z_{n}\right)-30 \sqrt{6} T_{R} \sin \left(\frac{8}{5} \sqrt{\frac{2}{3}} T_{R} Z_{n}\right)\right)}{4\left(25 \rho_{2}^{2}+24 T_{R}^{2}\right)} \delta \kappa_{0}+\delta T_{0 i} .
\end{aligned}
$$

The final timing jitter is obtained by integration of the last expression over the $N$ amplifiers stages

$$
\begin{aligned}
\left\langle\delta T_{0}^{2}(Z)\right\rangle= & \frac{100 T_{R}^{2} Z}{16 Z_{a}\left(25 \rho_{2}^{2}+24 T_{R}^{2}\right)^{2}}\left\langle\delta \eta_{0}^{2}\right\rangle+\frac{5625 \rho_{2}^{2} Z}{16 Z_{a}\left(25 \rho_{2}^{2}+24 T_{R}^{2}\right)^{2}}\left\langle\delta \kappa_{0}^{2}\right\rangle \\
& +Z\left\langle\delta T_{0 i}^{2}\right\rangle+\text { damped oscillatory terms. }
\end{aligned}
$$

This result shows that bandwidth-limited amplification reduces the growth of the timing jitter, which changes from a quintic power dependence on distance (in the absence of filtering) to a linear dependence. 
Fig. 3 shows timing jitter for 1ps soliton in presence of bandwidth-limited amplification. In this situation we see that the frequency fluctuations contribution is more important than amplitude fluctuations.

\section{Conclusions}

We have shown, using perturbation methods, that in ultrashort solitons transmission regime the total timing jitter increases with the quintic power of distance and with amplifier spacing. The main contribution to this timing jitter comes from intrapulse Raman scattering. Nevertheless, as in longer solitons regime, the bandwidth-limited amplification may reduce the growth of timing jitter which becomes linear dependent on the distance.

\section{References}

[1] Hasegawa A and Tappert F, Appl. Phys. Lett., 1973, V.23, 142-144.

[2] Karpman V I and Maslov E M, Sov. Phys. JEPT, 1977, V.46, N 2, 281.

[3] Gordon J P and Haus H A, Opt. Lett., 1986, V.11, 665.

[4] Georges T and Favre F, J. Opt. Soc. Am. B, 1993, V.10, N. 10, 1880.

[5] Essiambre R and Agrawal G, Opt. Comm., 1996, V.131, 274.

[6] Kodama Y and Hasegawa A, Opt. Lett., 1992, V.17, N 1, 31.

[7] Blow K J, Doran N J and Wood D, J. Opt. Soc. Am. B, 1988, V.5, N 6, 1301. 